\title{
A Systematic Review of the Association between Amyloid- $\beta$ and $\tau$ Pathology with Functional Connectivity Alterations in the Alzheimer Dementia Spectrum Utilizing PET Scan and rsfMRI
}

\author{
Seyede Anis Hasani ${ }^{a}$ Mahsa Mayeli ${ }^{a, b} \quad$ Mohammad Amin Salehi ${ }^{a, b}$ \\ Rezvan Barzegar Parizi ${ }^{a}$

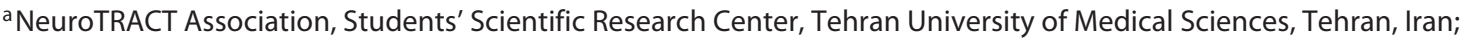 \\ ${ }^{\mathrm{b}} \mathrm{S} c h o o l$ of Medicine, Tehran University of Medical Sciences, Tehran, Iran
}

\section{Keywords \\ Alzheimer dementia · Amyloid- $\beta$ - Positron emission tomography scan $\cdot$ Resting-state functional magnetic resonance imaging $\cdot \tau$ protein}

\begin{abstract}
The association between functional connectivity $(\mathrm{FC})$ alterations with amyloid- $\beta(A \beta)$ and $\tau$ protein depositions in Alzheimer dementia is a subject of debate in the current literature. Although many studies have suggested a declining FC accompanying increased $A \beta$ and $\tau$ concentrations, some investigations have contradicted this hypothesis. Therefore, this systematic review was conducted to sum up the current literature in this regard. The PROSPERO guideline for systematic reviews was applied for development of a research protocol, and this study was initiated after getting the protocol approval. Studies were screened, and those investigating FC measured by resting-state functional MRI and $A \beta$ and $\tau$ protein depositions using amyloid and $\tau$ positron emission tomography were included. We categorized the included studies into 3 groups methodologically, addressing the question using global connectivity analysis (examining all regions of interest across the brain based on a functional atlas), seedbased connectivity analysis, or within-networks connectivity
\end{abstract}

analysis. The quality of the studies was assessed using the Newcastle-Ottawa Scale. Among 31 included studies, 14 found both positive and negative correlations depending on the brain region and stage of the investigated disease, while 7 showed an overall negative correlation, 8 indicated an overall positive correlation, and 2 found a nonsignificant association between protein deposition and FC. The investigated regions were illustrated using tables. The posterior default mode network, one of the first regions of amyloid accumulation, and the temporal lobe, the early $\tau$ deposition region, are the 2 most investigated regions where inconsistencies exist. In conclusion, our study indicates that transneuronal spreading of $\mathrm{t}$ and the amyloid hypothesis can justify higher FC related to higher protein depositions when global connectivity analysis is applied. However, the discrepancies observed when investigating the brain locally could be due to the varying manifestations of the amyloid and $\tau$ overload compensatory mechanisms in the brain at different stages of the disease with hyper- and hypoconnectivity cycles that can occur repeatedly. Nevertheless, further studies investigating both amyloid and $\tau$ deposition simultaneously while considering the stage of Alzheimer dementia are required to assess the accuracy of this hypothesis.

(c) 2021 The Author(s)

Published by S. Karger AG, Basel karger@karger.com www.karger.com/dee

Karger $\stackrel{\text { ' }}{5}$

BOPEN ACCESS
(C) 2021 The Author(s)

Published by S. Karger AG, Basel

This is an Open Access article licensed under the Creative Commons Attribution-NonCommercial-4.0 International License (CC BY-NC) (http://www.karger.com/Services/OpenAccessLicense), applicable to the online version of the article only. Usage and distribution for commercial purposes requires written permission.
Correspondence to:

Mahsa Mayeli,mahsamayelii@gmail.com 


\section{Introduction}

Alzheimer dementia (AD) manifests in a spectrum consisting of a long-lasting preclinical stage, a phase of mild cognitive impairment (MCI), and, eventually, fully manifested dementia [1]. Related pathophysiological changes primarily occur decades before the manifestation of cognitive decline symptoms [2]. Thus, the current focus is on early diagnosis and therapeutic approaches at the MCI and even preclinical disease stages to prevent or delay dementia onset. Although the use of imaging biomarkers including structural magnetic resonance imaging (MRI), amyloid positron emission tomography (PET), and 18F-fluorodeoxyglucose (FDG)$\mathrm{PET}$ is validated for diagnosis and prognosis at all stages of $\mathrm{AD}$ [3], functional connectivity (FC) measured by resting-state functional MRI (rsfMRI) promises a very early diagnosis in $\mathrm{AD}$ and other neurodegenerative diseases [4].

Extracellular deposits of amyloid- $\beta(\mathrm{A} \beta)$ peptides and intracellular neurofibrillary tangles of $\tau$ proteins are 2 important neuropathological hallmarks of $\operatorname{AD}[5,6]$. The correlation between these molecular hallmarks and alterations in FC remains a pivotal quest [7]. Regarding $\mathrm{A} \beta$, the earliest accumulation starts within the default mode network (DMN) [8]. While the higher amyloid burden has been correlated with a lower FC in the posterior cingulate cortex and precuneus (i.e., major hub regions in the DMN) in some works [9-11], other studies have linked $\mathrm{A} \beta$ to increased connectivity in DMN regions [12]. In addition to $\mathrm{DMN}$, an association between the global A $\beta$ load and aberrant FC is seen in other intrinsic connectivity networks [13-15]. Moreover, when applying a voxelwise approach globally rather than within networks, the correlation between $A \beta$ deposition and FC shows that a higher $A \beta$ deposition can be related to both increased and decreased FC in various brain regions [13].

Regarding $\tau$, some studies have found a positive association between FC and $\tau$ propagation, supporting the view of the trans-synaptic spread of $\tau$ across neuronal connections in $\mathrm{AD}[16,17]$ either at the global connectivity level $[18,19]$ or at the seed-to-voxel level [20]. However, other researchers have shown that, at a local level of $\tau$ deposition, a higher $\tau$ burden in some regions such as the posterior DMN (pDMN) and the inferior temporal cortex is related to declining connectivity $[21,22]$.

Altogether, these discrepant results concerning the association between $\tau$ and $A \beta$ deposition with alterations of FC and the difficulties of conducting original neuroimag- ing research urged us to systematically review the literature in this regard, aiming to interpret the related current literature comprehensively.

\section{Materials and Methods}

The present systematic review was prepared based on Preferred Reporting Items for Systematic reviews and Meta-Analyses (PRISMA) guidelines [23]. The study protocol was approved in PROSPERO with the registration code CRD42020154057 (https://www. crd.york.ac.uk/PROSPERO/).

\section{Search Strategy}

We searched the PubMed and Scopus Medical databases and used the Google Scholar search engine up to February 2021. The following search terms and combinations were used: "functional connectivity" OR "intrinsic connectivity" OR "connectome" OR "resting state") AND (tau OR amyloid) AND (normal OR healthy OR Alzheimer OR "mild cognitive impairment" OR preclinical OR prodromal OR "subjective memory complaint"), without any language or publication time restrictions. Then, 2 authors independently screened all of the titles and abstracts. The full texts of those studies found to be eligible for inclusion based on their abstracts were further studied and assessed for inclusion. Further discussions among all of the authors resolved disagreements regarding the inclusion of the studies.

\section{Inclusion and Exclusion Criteria}

The applied inclusion criteria were as follows: original studies using rsfMRI to examine FC while measuring amyloid or $\tau$ deposition or both by PET. Opinions, book chapters, reviews, letters, conference abstracts, protocols, and animal studies were excluded.

Following the initial screening, in the full-text assessment phase, studies applying any statistical method other than correlation and regression analysis for assessment of the alterations of FC were excluded. Also, we only included studies in which the following categorization was considered: amyloid-negative normals, amyloid-positive cognitively normals (preclinical $\mathrm{AD}$ ), subjective memory complaints $(\mathrm{SMC}), \mathrm{MCI}$ or prodromal $\mathrm{AD}$, and $\mathrm{AD}$ dementia.

\section{Data Extraction}

Two authors separately extracted the following data: bibliographic details of papers such as author name, publication year, study design, and demographic data of the participants, including the number of participants, age, gender, clinical inclusion, and exclusion criteria. Regarding rsfMRI and PET, we excluded some details such as: magnetic field strength, repetition time and echo time, connectivity analysis (e.g., seed based, whole brain, etc.), $\tau$-PET or amyloid-PET tracers, and the cutoff of amyloid-PET tracer uptake for amyloid positivity. Finally, the following details of the association analysis were extracted: (1)the type of association analysis (e.g., correlation or regression), (2) the name of the region(s) or network(s) with FC involved in the association, and (3) whether the amyloid or $\tau$ burden that was involved in the association was global or local and, if it was local, the name of the region(s).
Investigating the Association between $\mathrm{A} \beta$ and $\tau$ Pathology with FC Alterations in AD
Dement Geriatr Cogn Disord Extra 2021;11:78-90 DOI: $10.1159 / 000516164$ 


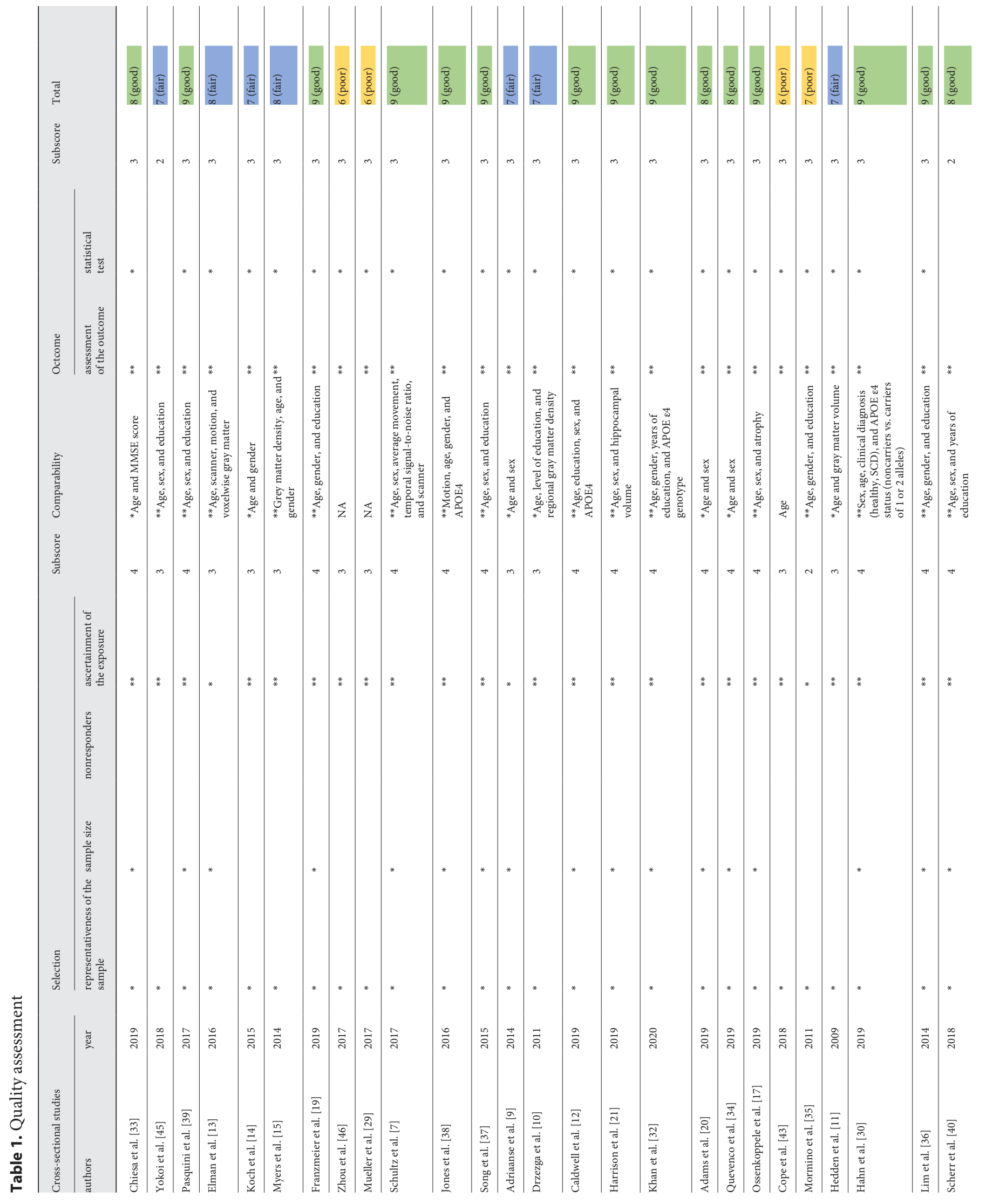




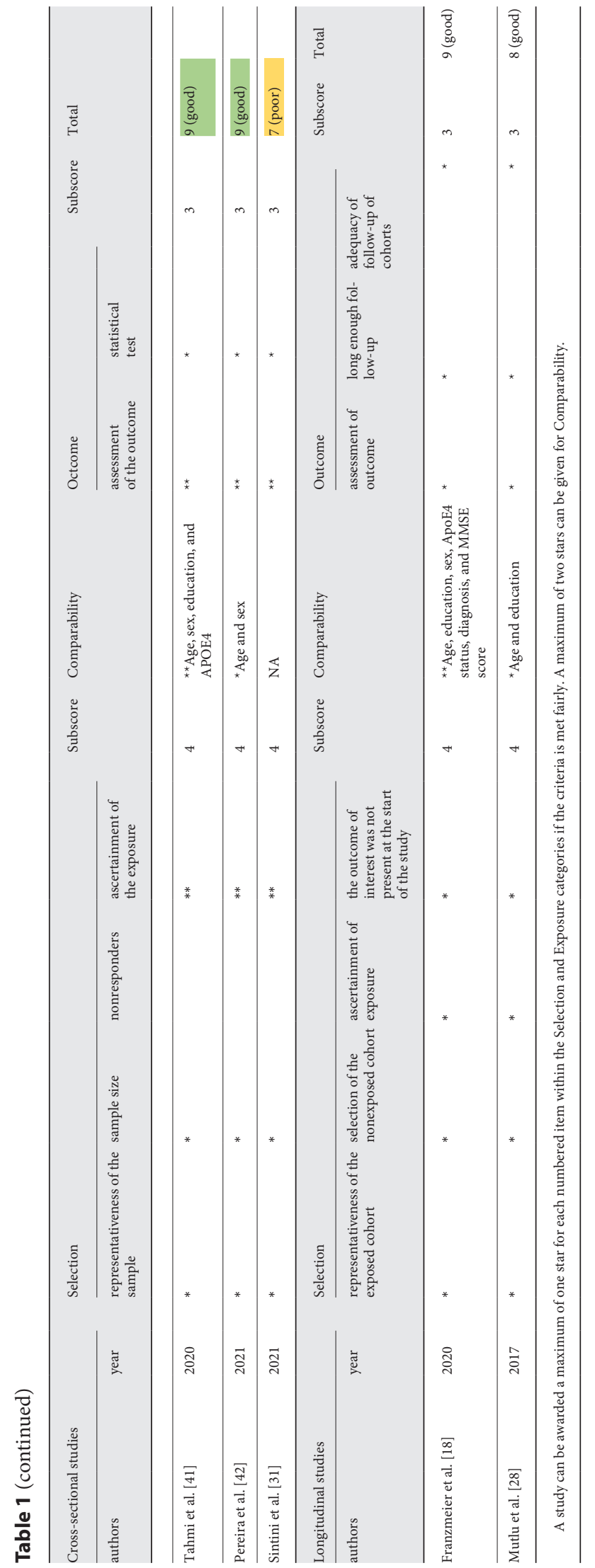

Investigating the Association between $\mathrm{A} \beta$ and $\tau$ Pathology with FC Alterations in AD
Quality Assessment

The quality of all of the included studies was assessed using a modified version of the Newcastle-Ottawa Scale (NOS) for observational studies. This scale rates the quality of studies in 3 major fields, i.e., selection, comparability, and outcome. A quality score ranging from 0 (minimum) to 10 (maximum) stars was allocated to each cross-sectional study, and for longitudinal studies a maximum score of 9 was considered. The definition of SMC, MCI, and AD dementia was based on the following criteria: for SMC, having a self-reported persistent memory decline assessed using the Cognitive Change Index and a normal cognitive performance (a normal WMS-LM delay recall performance, a normal MMSE, i.e., between 24 and 30, and a CDR score of 0) [24]; for MCI, Petersen criteria (MMSE $>24, \mathrm{CDR}=0.5$, and showing objective memory loss on the education-adjusted Wechsler Memory Scale-II but preserved activities of daily living) [25]; and for AD, National Institute on Aging research criteria for probable AD disease [26] (Table 1).

\section{Results}

\section{Study Selection and Characteristics}

A search of the PubMed database yielded 387 titles, while 609 records were identified through a search of Scopus, and 23,400 results were drawn from Google scholar. After extraction of those that did not fulfill the inclusion criteria and duplicates, 62 records were found to be eligible for full-text assessment. After application of the exclusion criteria, 31 studies were enrolled into this systematic review (Fig. 1; Table 2).

\section{Quality of Studies}

Study quality was rated as good, fair, or poor based on thresholds described in the Agency for Healthcare Research and Quality (AHRQ) standards [27]. Of the 31 included studies, 29 were cross-sectional and 2 had a longitudinal design. Regarding the sample size assessment of the cross-sectional studies, those with $<50$ participants received no star (9 studies). None of the included studies displayed a response rate or described the characteristics of the responders and the nonresponders. We allocated 2 stars to studies that measured FC using a 3-T scanner and 1 star to studies that used a 1.5 - $\mathrm{T}$ scanner ( 3 studies) to ascertain exposure. In the comparability section, age and sex were considered as the 2 most important confounding factors. We allocated only 1 star to studies that controlled their analysis for these 2 factors ( 8 studies). If a study used other factors, another star was allocated (17 studies). Four studies received no star in this part. In the outcome section, all of the included studies had an independent blind assessment, and only 2 studies did not present a $p$ value for their correlation analysis. Overall, from 29 cross-sec- 
Fig. 1. Study selection flowchart.

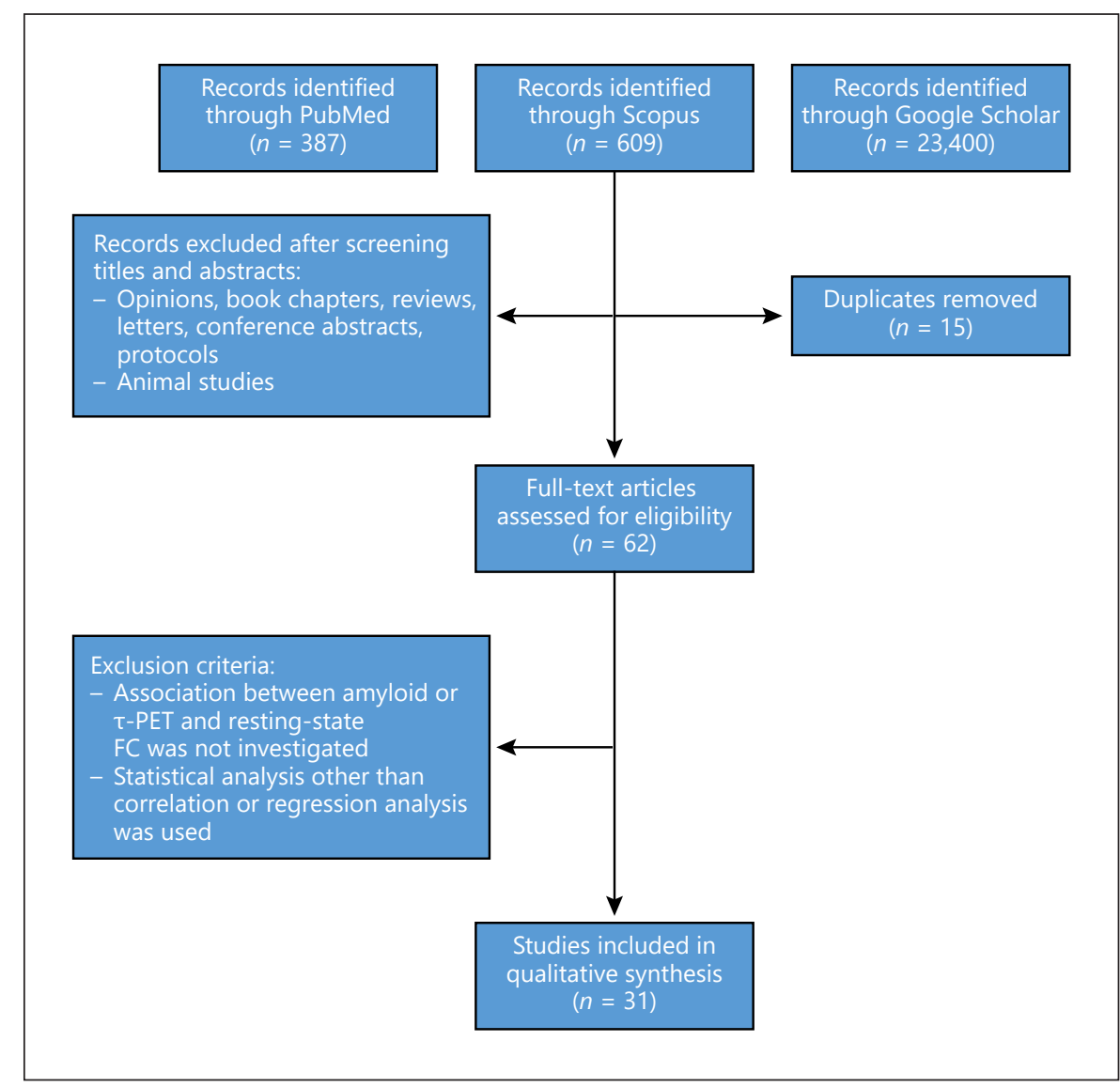

Table 2. Summary of the results

\begin{tabular}{llll}
\hline & $\begin{array}{l}\text { Studies } \\
\text { reporting a positive } \\
\text { association, } n\end{array}$ & $\begin{array}{l}\text { Studies } \\
\text { reporting a negative } \\
\text { association, } n\end{array}$ & $\begin{array}{l}\text { Studies } \\
\text { reporting both positive and } \\
\text { negative associations, } n\end{array}$ \\
\hline $\begin{array}{l}\text { Association between amyloid PET and FC } \\
\text { Association between } \tau \text {-PET and FC }\end{array}$ & 8 & 6 & 9 \\
\hline
\end{tabular}

tional studies, 17 studies received good, 7 studies received fair, and 5 studies received poor ratings. Considering longitudinal studies, both of them were deemed to be of good quality.

\section{Association between Amyloid PET and FC}

Studies investigating the alterations of FC concerning amyloid deposits have addressed the question through global connectivity, seed-based connectivity, or withinnetwork connectivity (Table 3 ).

While examining the global connectivity with all regions of interest (ROI) across the brain, 1 study indicated a positive overall association between connectivity in healthy elderly subjects and the baseline global $A \beta$ burden in patients at the MCI and AD stages [28]. However, another study suggested both positive and negative associations based on graph analysis [29]. Two other studies reported a positive association $[30,31]$, while 1 had applied dynamic connectivity [30]. When static connectivity was used, a nonsignificant association was found [30].

Seed-based connectivity was investigated either through all voxels and ROI across the brain or within known networks. In 6 studies applying the first approach, 3 studies indicated a negative correlation between global 


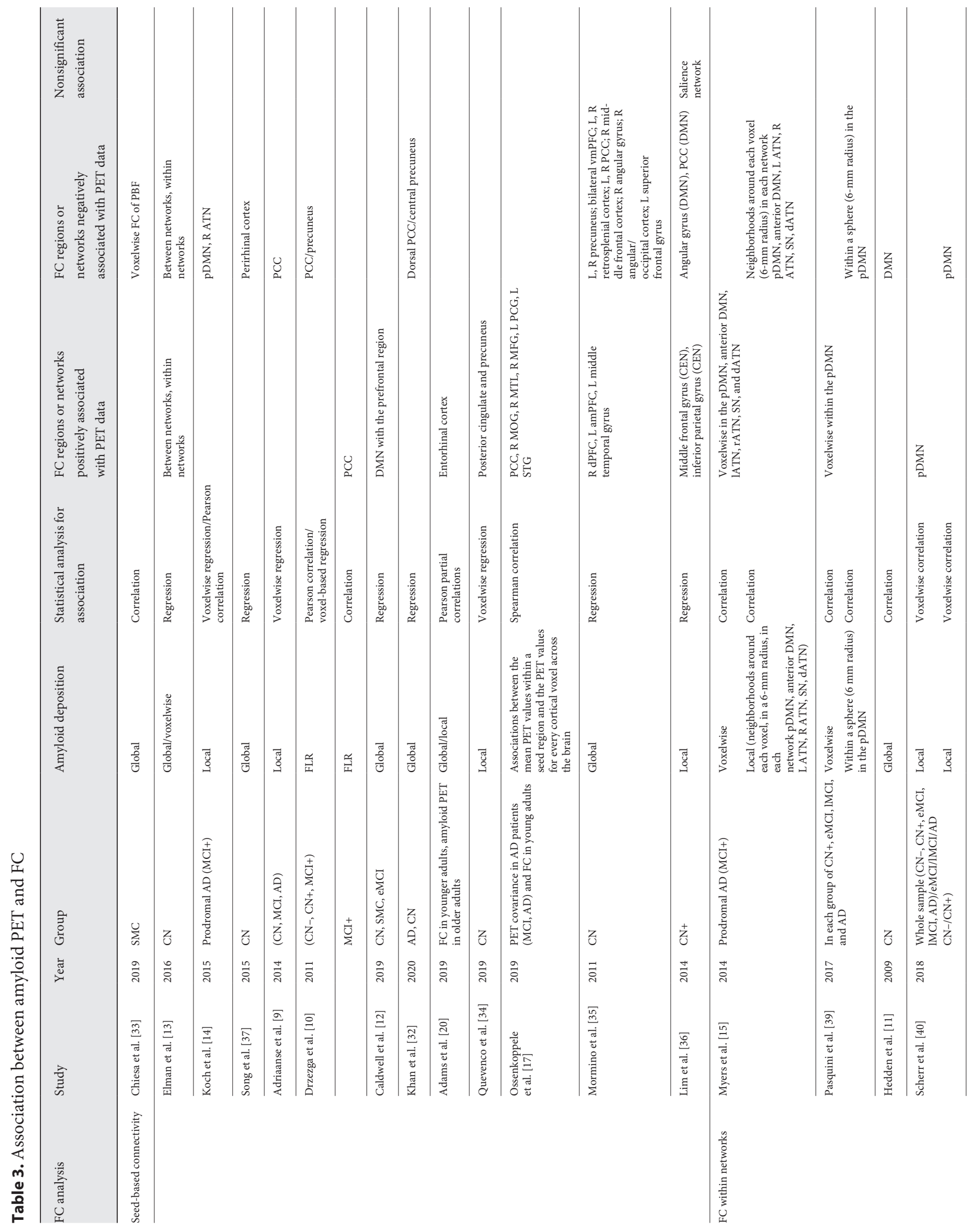




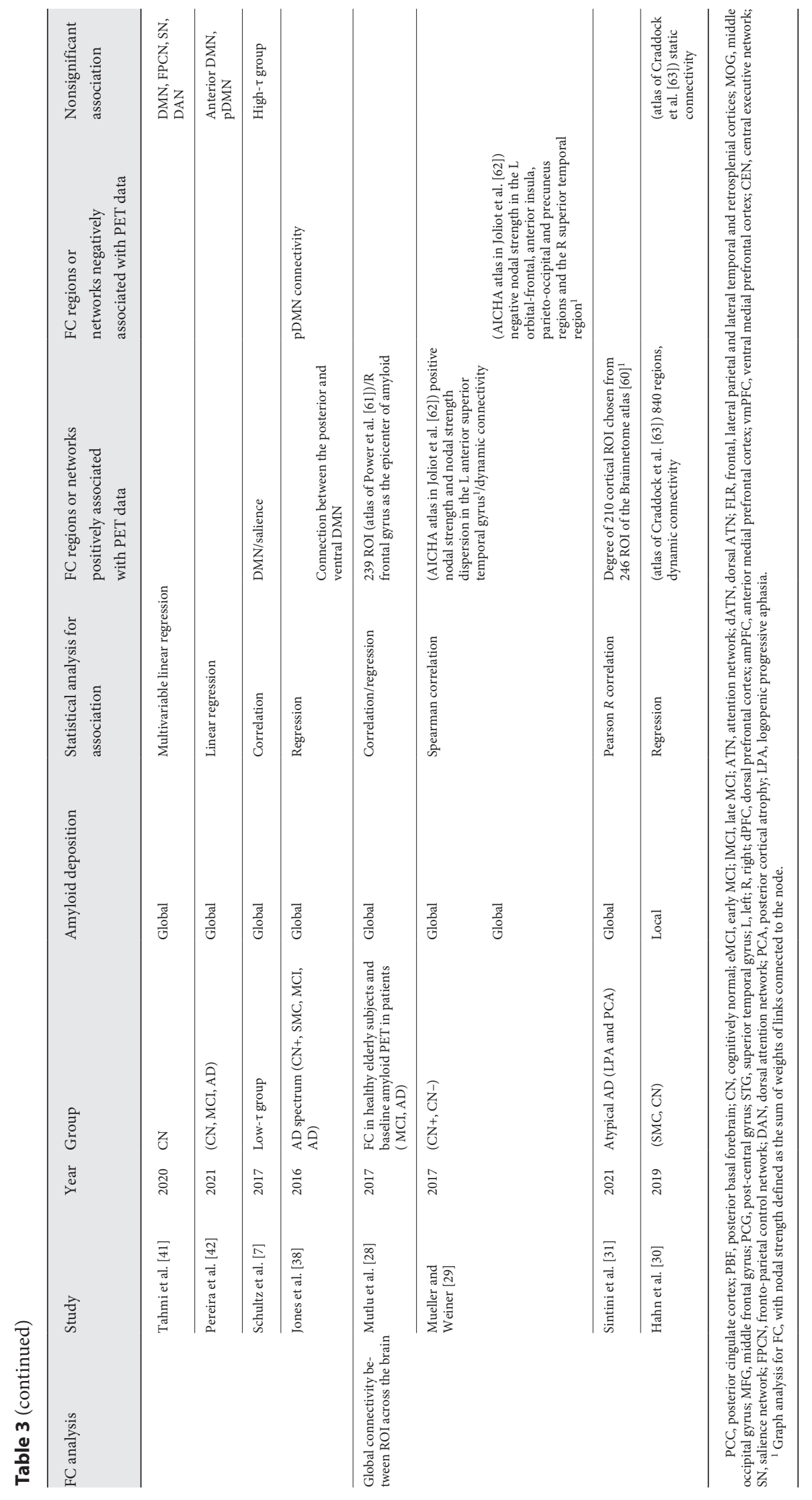


$\mathrm{A} \beta$ depositions and connectivity in $\mathrm{AD}$ patients and $\mathrm{CN}$ participants [32], all $\mathrm{CN}-, \mathrm{CN}+$, and MCI subjects [10], and SMC participants [33], among which 1 work also found a positive correlation when conducting the analysis merely in MCI patients [10]. Two other studies indicated a positive association between $\mathrm{A} \beta$ deposition and FC $[17$, $20]$. The connectivity changes associated with local $A \beta$ depositions were merely investigated in 1 study, which yielded a positive association [34].

Out of 7 studies applying the second approach, 3 studies suggested both higher and lower FC related to a higher global or local amyloid deposition $[13,35,36]$. Of the other 4 studies, 1 found increased FC between the left prefrontal cortex and the anterior DMN while the amyloid deposition increased globally [12]. The other 3 studies reported a decreased FC; 2 were related to the local amyloid burden $[9,14]$; and 1 was related to the global amyloid burden [37].

FC within networks in association with $\mathrm{A} \beta$ was explored in 8 studies. While studying amyloid deposition globally, 1 work suggested a positive association, [7], 1 suggested a negative association [11], and another suggested both a positive and a negative association [38]. Two other studies revealed a positive association, using the voxelwise approach, for the correlation between FC and amyloid deposition. They also suggested a negative association when the correlation analysis was conducted locally on the scale of a 6 -mm radius sphere $[15,39]$. Using the voxelwise approach, another study showed both positive and negative associations [40]. Finally, 2 studies reported a nonsignificant correlation between amyloid PET and FC [41, 42].

Association between $\tau$-PET and FC

In the association between FC and $\tau$-PET, 3 approaches were utilized to investigate FC, i.e., examination of all ROI across the brain based on functional atlases, seedbased connectivity analysis, and exploration of FC within networks (Table 4).

While investigating global connectivity in association with $\tau$, both studies suggested a positive association [18, 19]. Moreover, of the 2 other studies that used graph analysis for FC, one indicated both positive and negative correlations [43] and another reported a negative correlation [31].

Of the 4 studies applying seed-based connectivity, 2 indicated a positive association $[20,44]$ while 1 indicated a lower hippocampal FC in association with a higher $\tau$ burden in the inferior temporal cortex [21]. The last study mainly found a higher FC related to $\tau$-PET covariance [17].

Exploring FC within networks in association with $\tau$, one study observed a positive correlation in early AD patients and a negative association in $\mathrm{CN}$ - cases [45]. Another study suggested no association between global $\tau$-PET and FC in the anterior DMN or the pDMN [42]. Investigation of local $\tau$ deposition yielded positive results in 2 studies $[7,46]$, and merely 1 prior work suggested a negative association when only $\mathrm{CN}+$ cases were examined [7]. Finally, 1 recent work exploring $\tau$-PET longitudinally revealed a higher FC-to- $\tau$ change in 7 brain networks [18].

\section{Discussion}

This study was conducted to systematically explore the current literature regarding the association between $A \beta$ and $\tau$ deposition with FC alterations in the AD spectrum utilizing $A \beta$ and $\tau$-PET scan and rsfMRI. A significant body of literature has already been dedicated to identifying AD hallmarks through biomarkers and neuroimaging signature regions [47]; however, specifying structural and FC pattern alterations regarding $A \beta$ and $\tau$ depositions remains controversial $[22,48,49]$. Recent findings attributing the neuropsychiatric symptoms of $\mathrm{AD}$ to connectivity alterations have signified that further studies are addressing this question [50].

Herein, we categorized the FC alteration analysis into the following 3 groups: global, seed-based, or within the network. Also, the following 3 categories of analysis were considered for $\tau$-PET or amyloid PET scan: locally, globally, or in a voxelwise method.

Early works addressing this question regarding the correlation between amyloid and FC have suggested a hypoconnectivity accompanying amyloid deposition [10, $11,51,52]$. However, this speculation was later challenged by studies indicating hyperconnectivity in both preclinical and clinical phases of the disease $[13,15,35,39]$. The observed hyperconnectivity is speculated to be a system level compensatory mechanism to maintain function [7]. This hyperconnectivity possesses certain features, including region specificity, particularly regarding the pDMN $[22,44]$. A particular pattern correlating with the disease stage was noted in some studies [38]. Eventually, the overwhelming pathology mechanism is indicted for altering this hyperconnectivity to hypoconnectivity as the disease progresses [53]. 


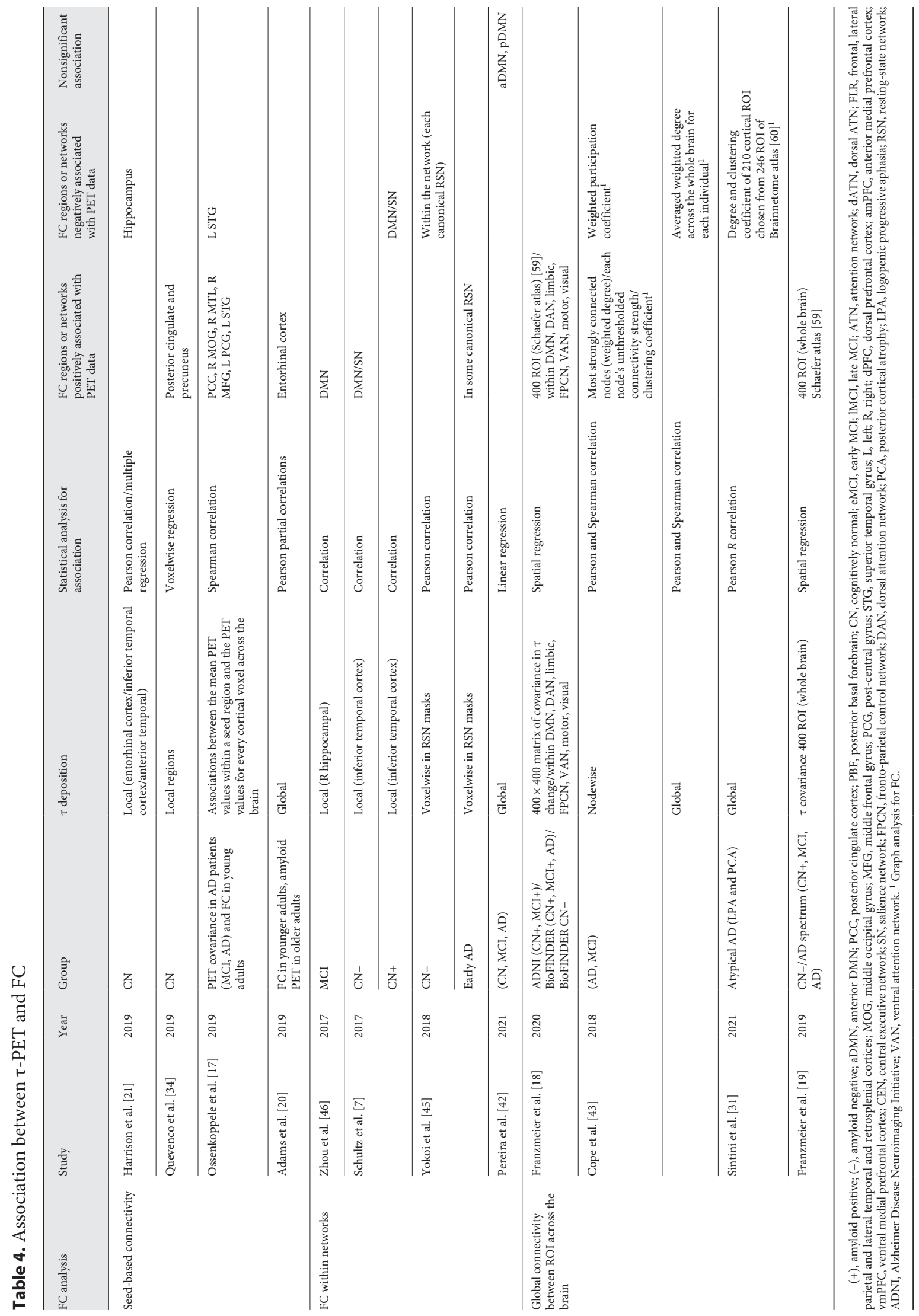


The majority of works included in this study that evaluated FC alterations concerning $\tau$ deposition have found increased connectivity, which is consistent with the "trans-synaptic spread" model that proposing that, since $\tau$ proteins are transmitted through synaptic connections, its spreading pattern resembles the FC patterns across the brain $[17,19,43]$. This phenomenon is not influenced by the presence of amyloid or cognitive impairment $[19,20]$. However, the lower FC related to a higher $\tau$ deposition has also been reported when the connectivity of the hippocampus, the critical component of the medial temporal lobe memory system, is considered. A higher $\tau$ burden in the anterior temporal regions is associated with functional disconnectivity between the hippocampus and other components of the medial temporal lobe (MTL) memory system, which predicts memory decline [21].

According to those works studying the amyloid, $\tau$, and FC alterations altogether, when the age-related $\tau$ accumulation increases in MTL, the processing pressure will increase in brain networks. A compensatory shift may occur toward the pDMN, leading to a hyperconnectivity within it. In this stage, if the brain does not have a compensatory ability, the overloading phenomena are speculated to later cause the observed decreased connectivity in the $\mathrm{pDMN}$ and hyperconnectivity regarding other brain regions, particularly with the frontal lobe. Amyloid plaques are assumed to be subsequently created in response to the synapses' noisiness and mediate and facilitate the connections between $\tau$ deposition and the largescale brain networks $[20,22]$. Thus, the early amyloid accumulation phase is associated with a higher FC in the DMN and other hubs [7, 22], and it seems that this higher FC guides more and more spreading of $\tau$, which later again decreases the FC [20]. If no compensatory mechanism atones this cycle, then neurodegeneration occurs [22]. The phase of hyperconnectivity continues until $\tau$ spreads to the neocortical regions [7]. Spreading of $\tau$ to the neocortical regions rarely happens in the absence of an amyloid burden, while in $\mathrm{A} \beta$-positive individuals the $\tau$ pathology spreads to the neocortex [54]. Increasing $\tau$ in the inferior temporal cortex, as a neocortical region in which early $\tau$ propagation to the neocortex occurs, is associated with DMN hypoconnectivity in $A \beta$-positive healthy adults. In contrast, in $A \beta$-negative individuals, DMN hyperconnectivity related to $\tau$ has been observed [7].

As a total interpretation, amyloid pathology could be associated with hyperconnectivity, which is assumed as a signal for compensatory activity if $\tau$ has not yet propagated to the neocortex, but the spread of $\tau$ in neocortical regions leads to a loss of FC [54]. It may suggest that both a high amyloid deposition and a high $\tau$ deposition are unlikely to leave an individual clinically healthy [55]. The issue that should be considered concerning hyperconnectivity is whether compensation is a correct interpretation for increased FC [56]. An important study proposed another explanation, stating the hyperconnectivity may indicate a reduction in the dynamicity of FC in networks [7]. Moreover, the work by Elman et al. [13] demonstrated that an amyloid-related higher FC might reflect a decrease in anticorrelation between networks. Therefore, to have a more secure conclusion about the compensatory interpretation of hyperconnectivity, the association between increased FC and a better cognitive performance in individuals should be investigated [56].

\section{Conclusion}

We conclude that most controversies detected when investigating the connectivity alterations accompanying amyloid and $\tau$ depositions might primarily be due to methodological variances. That is to say, when studying the connectivity alterations globally (between ROI or voxels across the brain), a positive correlation is detected. This positive correlation is hypothesized to be caused by the transneuronal spreading pattern of both amyloid and $\tau$ proteins. However, while examining FC locally (in a network or even on a smaller scale), the positivity or negativity of the association depends on the stage of the disease. This is due to the fact that $A \beta$ and $\tau$ overload urges compensatory mechanisms that manifest as potentially repeatedly occurring hyper- and hypoconnectivity cycles, which mainly occur in the pDMN and other hub regions.

\section{Significance of the Study}

Identification of distinct network features is of recent interest for early diagnosis and prognosis determination of neurological disorders. Among these are the connectivity alterations in different stages of $\mathrm{AD}$ [57]. Therefore, our study was conducted to recognize the specific connectivity alterations with $\tau$ and amyloid depositions, which might help to develop a network alteration pattern for early $\mathrm{AD}$ diagnosis and help to elucidate the network structure of the disease. 


\section{Future Directions}

The recently developed $\tau$ PET tracers [58] have left many fundamental questions regarding the correlation between $\tau$ pathology and brain networks unanswered. More multimodal cross-sectional and longitudinal studies will be needed to illuminate the correlation and interaction between functional brain connectivity and $\mathrm{AD}$ pathogenic proteins. All 3 measures, i.e., amyloid and $\tau$-PET and rs-fMRI, are to be investigated in all AD spectrum stages. Studying networks other than the DMN, connectivity between networks, and the dynamic feature of brain networks concerning both $\tau$ and amyloid deposition are pivotal quests to pursue $[13,30]$. Eventually, the actuality of the compensatory role of hyperconnectivity is also to be assessed by examining whether higher FC is correlated with a more successful cognitive performance [56].

\section{Statement of Ethics}

As this study is a literature review and there was no direct contact with human or animal cases, no informed consent was obtainable. The methodology was approved by the PROSPERO community before the initiation of this study.

\section{Conflict of Interest Statement}

The authors have no conflict of interests to declare.

\section{Funding Sources}

No funding was received for this systematic review.

\section{Author Contributions}

S.A.H. conceived and designed the evaluation and drafted this paper. M.M. participated in designing the evaluation, performed parts of the analysis, and drafted this paper. M.A.S. reevaluated the data and revised this paper. R.B.P. helped to interpret the data and revise this paper. All of the authors read and approved the final version of this work.

\section{References}

1 Sperling RA, Aisen PS, Beckett LA, Bennett DA, Craft S, Fagan AM, et al. Toward defining the preclinical stages of Alzheimer's disease: recommendations from the National Institute on Aging-Alzheimer's Association workgroups on diagnostic guidelines for Alzheimer's disease. Alzheimers Dement. 2011 May; $7(3): 280-92$.

2 Jones DT, Lowe VJ, Wiste HJ, Senjem ML, Radford JG, Boeve BF, et al. Network-Based Tau Deposition Patterns Are Related to Functional Network Failure Largely Via Beta-Amyloid Across the Alzheimer's Spectrum. Alzheimers Dement. 2016 Jul;12(7):502.

3 Albert MS, DeKosky ST, Dickson D, Dubois B, Feldman HH, Fox NC, et al. The diagnosis of mild cognitive impairment due to $\mathrm{Al}-$ zheimer's disease: recommendations from the National Institute on Aging-Alzheimer's Association workgroups on diagnostic guidelines for Alzheimer's diseas. Alzheimers Dement. 2011 May;7(3):270-9.

4 Jalilianhasanpour R, Beheshtian E, Sherbaf G, Sahraian S, Sair HI. Functional Connectivity in Neurodegenerative Disorders: Alzheimer's Disease and Frontotemporal Dementia. Top Magn Reson Imaging. 2019 Dec;28(6):31724.

5 Braak H, Braak E, Bohl J. Staging of Alzheimer-related cortical destruction. Eur Neurol. 1993;33(6):403-8.
6 Hyman BT, Phelps CH, Beach TG, Bigio EH, Cairns NJ, Carrillo MC, et al. National Institute on Aging-Alzheimer's Association guidelines for the neuropathologic assessment of Alzheimer's disease. Alzheimers Dement. 2012 Jan;8(1):1-13.

7 Schultz AP, Chhatwal JP, Hedden T, Mormino EC, Hanseeuw BJ, Sepulcre J, et al. Phases of Hyperconnectivity and Hypoconnectivity in the Default Mode and Salience Networks Track with Amyloid and Tau in Clinically Normal Individuals. J Neurosci. 2017 Apr; 37(16):4323-31.

8 Palmqvist S, Schöll M, Strandberg O, Mattsson N, Stomrud E, Zetterberg H, et al. Earliest accumulation of $\beta$-amyloid occurs within the default-mode network and concurrently affects brain connectivity. Nat Commun. 2017 Oct;8(1):1214.

9 Adriaanse SM, Sanz-Arigita EJ, Binnewijzend MA, Ossenkoppele R, Tolboom N, van Assema DM, et al. Amyloid and its association with default network integrity in Alzheimer's disease. Hum Brain Mapp. 2014 Mar;35(3): 779-91.

10 Drzezga A, Becker JA, Van Dijk KR, Sreenivasan A, Talukdar T, Sullivan C, et al. Neuronal dysfunction and disconnection of cortical hubs in non-demented subjects with elevated amyloid burden. Brain. 2011 Jun;134(Pt 6): $1635-46$.
11 Hedden T, Van Dijk KR, Becker JA, Mehta A, Sperling RA, Johnson KA, et al. Disruption of functional connectivity in clinically normal older adults harboring amyloid burden. J Neurosci. 2009 Oct;29(40):12686-94.

12 Caldwell JZ, Zhuang X, Leavitt MJ, Banks SJ, Cummings J, Cordes D; Alzheimer's Disease Neuroimaging Initiative. Sex moderates amyloid and apolipoprotein $\varepsilon 4$ effects on default mode network connectivity at rest. Front Neurol. 2019 Aug;10(AUG):900.

13 Elman JA, Madison CM, Baker SL, Vogel JW, Marks SM, Crowley S, et al. Effects of BetaAmyloid on Resting State Functional Connectivity Within and Between Networks Reflect Known Patterns of Regional Vulnerability. Cereb Cortex. 2016 Feb;26(2):695-707.

14 Koch K, Myers NE, Göttler J, Pasquini L, Grimmer T, Förster S, et al. Disrupted intrinsic networks link amyloid- $\beta$ pathology and impaired cognition in prodromal Alzheimer's disease. Cereb Cortex. 2015 Dec;25(12): 4678-88.

15 Myers N, Pasquini L, Göttler J, Grimmer T, Koch K, Ortner M, et al. Within-patient correspondence of amyloid- $\beta$ and intrinsic network connectivity in Alzheimer's disease. Brain. 2014 Jul;137(Pt 7):2052-64. 
16 Kim HR, Lee P, Seo SW, Roh JH, Oh M, Oh JS, et al. Comparison of amyloid $\beta$ and tau spread models in Alzheimer's disease. Cereb Cortex [Internet]. 2018 [cited 2019 Jul 4]. Available from: https://academic.oup.com/ cercor/advance-article/doi/10.1093/cercor/ bhy311/5253203.

17 Ossenkoppele R, Iaccarino L, Schonhaut DR, Brown JA, La Joie R, O’Neil JP, et al. Tau covariance patterns in Alzheimer's disease patients match intrinsic connectivity networks in the healthy brain. Neuroimage Clin. 2019; 23:101848.

18 Franzmeier N, Neitzel J, Rubinski A, Smith R, Strandberg O, Ossenkoppele R, et al.; Alzheimer's Disease Neuroimaging Initiative (ADNI). Functional brain architecture is associated with the rate of tau accumulation in Alzheimer's disease. Nat Commun. 2020 Jan; 11(1):347.

19 Franzmeier N, Rubinski A, Neitzel J, Kim Y, Damm A, Na DL, et al.; Alzheimer's Disease Neuroimaging Initiative. Functional connectivity associated with tau levels in ageing, Alzheimer's, and small vessel disease. Brain. 2019 Apr;142(4):1093-107.

20 Adams JN, Maass A, Harrison TM, Baker SL, Jagust WJ, Wills H, et al. Cortical tau deposition follows patterns of entorhinal functional connectivity in aging. Elife. 2019 Sep; 8:e49132.

21 Harrison TM, Maass A, Adams JN, Du R, Baker SL, Jagust WJ. Tau deposition is associated with functional isolation of the hippocampus in aging. Nat Commun. 2019 Oct; 10(1):4900.

22 Jones DT, Graff-Radford J, Lowe VJ, Wiste HJ, Gunter JL, Senjem ML, et al. Tau, amyloid, and cascading network failure across the Alzheimer's disease spectrum. Cortex. 2017 Dec;97:143-59.

23 Moher D, Liberati A, Tetzlaff J, Altman DG; PRISMA Group. Preferred reporting items for systematic reviews and meta-analyses: the PRISMA statement. Ann Intern Med. 2009 Aug;151(4):264-9.

24 Jessen F, Amariglio RE, van Boxtel M, Breteler M, Ceccaldi M, Chételat G, et al.; Subjective Cognitive Decline Initiative (SCD-I) Working Group. A conceptual framework for research on subjective cognitive decline in preclinical Alzheimer's disease. Alzheimers Dement. 2014 Nov;10(6):844-52.

25 Petersen RC, Caracciolo B, Brayne C, Gauthier S, Jelic V, Fratiglioni L. Mild cognitive impairment: a concept in evolution. J Intern Med. 2014 Mar;275(3):214-28.

26 McKhann GM, Knopman DS, Chertkow H, Hyman BT, Jack CR Jr, Kawas CH, et al. The diagnosis of dementia due to Alzheimer's disease: recommendations from the National Institute on Aging-Alzheimer's Association workgroups on diagnostic guidelines for Alzheimer's disease. Alzheimers Dement. 2011 May;7(3):263-9.
27 McPheeters ML, Kripalani S, Peterson NB, Idowu RT, Jerome RN, Potter SA AJ. Closing the quality gap: revisiting the state of the science (vol. 3: quality improvement interventions to address health disparities). Evid Rep Technol Assess (Full Rep). 2012 Aug;(208.3): $1-475$.

28 Mutlu J, Landeau B, Gaubert M, de La Sayette V, Desgranges B, Chételat G. Distinct influence of specific versus global connectivity on the different Alzheimer's disease biomarkers. Brain. 2017 Dec;140(12):3317-28.

29 Mueller SG, Weiner MW. Amyloid associated intermittent network disruptions in cognitively intact older subjects: structural connectivity matters. Front Aging Neurosci. 2017 Dec;9(DEC):418.

30 Hahn A, Strandberg TO, Stomrud E, Nilsson $M$, van Westen D, Palmqvist S, et al. Association Between Earliest Amyloid Uptake and Functional Connectivity in Cognitively Unimpaired Elderly. Cereb Cortex. 2019 May; 29(5):2173-82.

31 Sintini I, Graff-radford J, Jones DT, Botha H, Martin PR, Machulda MM, et al. Tau and Amyloid Relationships with Resting-state Functional Connectivity in Atypical Alzheimer's Disease. Cereb Cortex. 2021 Feb; 31(3):1693-706.

32 Khan W, Amad A, Giampietro V, Werden E, De Simoni S, O’Muircheartaigh J, et al.; Alzheimer's Disease Neuroimaging Initiative. The heterogeneous functional architecture of the posteromedial cortex is associated with selective functional connectivity differences in Alzheimer's disease. Hum Brain Mapp. 2020 Apr;41(6):1557-72.

33 Chiesa PA, Cavedo E, Vergallo A, Lista S, Potier MC, Habert MO, et al.; INSIGHT-preAD study group; Alzheimer Precision Medicine Initiative (APMI). Differential default mode network trajectories in asymptomatic individuals at risk for Alzheimer's disease. Alzheimers Dement. 2019 Jul;15(7):940-50.

34 Quevenco FC, Schreiner SJ, Preti MG, van Bergen JM, Kirchner T, Wyss M, et al. GABA and glutamate moderate beta-amyloid related functional connectivity in cognitively unimpaired old-aged adults. Neuroimage Clin. 2019;22:101776.

35 Mormino EC, Smiljic A, Hayenga AO, Onami $\mathrm{SH}$, Greicius MD, Rabinovici GD, et al. Relationships between $\beta$-amyloid and functional connectivity in different components of the default mode network in aging. Cereb Cortex. 2011 Oct;21(10):2399-407.

36 Lim HK, Nebes R, Snitz B, Cohen A, Mathis $\mathrm{C}$, Price J, et al. Regional amyloid burden and intrinsic connectivity networks in cognitively normal elderly subjects. Brain. 2014 Dec; 137(Pt 12):3327-38.

37 Song Z, Insel PS, Buckley S, Yohannes S, Mezher A, Simonson A, et al. Brain amyloid- $\beta$ burden is associated with disruption of intrinsic functional connectivity within the medial temporal lobe in cognitively normal elderly. J Neurosci. 2015 Feb;35(7):3240-7.
38 Jones DT, Knopman DS, Gunter JL, GraffRadford J, Vemuri P, Boeve BF, et al.; Alzheimer's Disease Neuroimaging Initiative. Cascading network failure across the $\mathrm{Al}$ zheimer's disease spectrum. Brain. 2016 Feb; 139(Pt 2):547-62.

39 Pasquini L, Benson G, Grothe MJ, Utz L, Myers NE, Yakushev I, et al.; Alzheimer's Disease Neuroimaging Initiative. Individual Correspondence of Amyloid- $\beta$ and Intrinsic Connectivity in the Posterior Default Mode Network Across Stages of Alzheimer's Disease. J Alzheimers Dis. 2017;58(3):763-73.

40 Scherr M, Pasquini L, Benson G, Nuttall R, Gruber M, Neitzel J, et al.; Alzheimer's Disease Neuroimaging Initiative. Decoupling of Local Metabolic Activity and Functional Connectivity Links to Amyloid in Alzheimer's Disease [Internet]. J Alzheimers Dis. 2018;64(2):405-15.

41 Tahmi M, Rippon B, Palta P, Soto L, Ceballos F, Pardo M, et al. Brain Amyloid Burden and Resting-State Functional Connectivity in Late Middle-Aged Hispanics. Front Neurol. 2020 Oct;11(October):529930.

42 Pereira JB, Janelidze S, Ossenkoppele R, Kvartsberg H, Brinkmalm A, Mattsson-Carlgren $\mathrm{N}$, et al. Untangling the association of amyloid- $\beta$ and tau with synaptic and axonal loss in Alzheimer's disease. Brain. 2021 Feb; 144(1):310-24.

43 Cope TE, Rittman T, Borchert RJ, Jones PS, Vatansever D, Allinson K, et al. Tau burden and the functional connectome in Alzheimer's disease and progressive supranuclear palsy. Brain. 2018 Feb;141(2):550-67.

44 Quevenco FC, van Bergen JM, Treyer V, Studer ST, Kagerer SM, Meyer R, et al. Functional Brain Network Connectivity Patterns Associated With Normal Cognition at Old-Age, Local $\beta$-amyloid, Tau, and APOE4. Front Aging Neurosci. 2020 Mar;12:46.

45 Yokoi T, Watanabe $\mathrm{H}$, Yamaguchi H, Bagarinao $\mathrm{E}$, Masuda $\mathrm{M}$, Imai $\mathrm{K}$, et al. Involvement of the precuneus/posterior cingulate cortex is significant for the development of Alzheimer's disease: A PET (THK5351, PiB) and resting fMRI study. Front Aging Neurosci. 2018 Oct; 10 (OCT):304.

46 Zhou Y, Bai B. Tau and Pet/Mri Imaging Biomarkers for Detecting and Diagnosing Early Dementia. Jacobs J Med diagnosis Med imaging. 2017 Nov;2(1):017.

47 Mayeli M, Mirshahvalad SM, Aghamollaii V, Tafakhori A, Abdolalizadeh A, Rahmani F. Plasma Neurofilament Light Chain Levels Are Associated With Cortical Hypometabolism in Alzheimer Disease Signature Regions. J Neuropathol Exp Neurol. 2019, Online ahead of print.

48 Shigemoto Y, Sone D, Maikusa N, Okamura $\mathrm{N}$, Furumoto S, Kudo Y, et al. Association of deposition of tau and amyloid- $\beta$ proteins with structural connectivity changes in cognitively normal older adults and Alzheimer's disease spectrum patients. Brain Behav. 2018 Dec; 8(12):e01145
Investigating the Association between $\mathrm{A} \beta$ and $\tau$ Pathology with FC Alterations in AD
Dement Geriatr Cogn Disord Extra 2021;11:78-90 DOI: $10.1159 / 000516164$ 
49 Li K, Luo X, Zeng Q, Jiaerken Y, Xu X, Huang $\mathrm{P}$, et al. Aberrant functional connectivity network in subjective memory complaint individuals relates to pathological biomarkers. Transl Neurodegener. 2018 Oct 19;7:27.

50 Balthazar ML, Pereira FR, Lopes TM, da Silva EL, Coan AC, Campos BM, et al. Neuropsychiatric symptoms in Alzheimer's disease are related to functional connectivity alterations in the salience network. Hum Brain Mapp. 2014 Apr;35(4):1237-46.

51 Sheline YI. Resting State Review in AD. 2014; 74(5):340-7.

52 Yi D, Choe YM, Byun MS, Sohn BK, Seo EH, Han J, et al. Differences in functional brain connectivity alterations associated with cerebral amyloid deposition in amnestic mild cognitive impairment. Front Aging Neurosci. 2015 Feb;7(FEB): 15

53 Brier MR, Thomas JB, Snyder AZ, Benzinger TL, Zhang D, Raichle ME, et al. Loss of intranetwork and internetwork resting state functional connections with Alzheimer's disease progression. J Neurosci. 2012 Jun;32(26): 8890-9.
54 van der Kant R, Goldstein LS, Ossenkoppele R. Amyloid- $\beta$-independent regulators of tau pathology in Alzheimer disease [Internet]. Nat Rev Neurosci. 2020 Jan;21(1):21-35.

55 Jack CR, Bennett DA, Blennow K, Carrillo MC, Feldman HH, Frisoni GB, et al. A/T/N: An unbiased descriptive classification scheme for Alzheimer disease biomarkers. Neurology. 2016 Aug;87(5):539-47.

56 Cabeza R, Albert M, Belleville S, Craik FI, Duarte A, Grady CL, et al. Maintenance, reserve and compensation: the cognitive neuroscience of healthy ageing. Nat Rev Neurosci. 2018 Nov; 19(11):701-10.

57 Stam CJ. Modern network science of neurological disorders. Nat Rev Neurosci. 2014 Oct; 15(10):683-95.

58 Saint-Aubert L, Lemoine L, Chiotis K, Leuzy A, Rodriguez-Vieitez E, Nordberg A. Tau PET imaging: present and future directions. Mol Neurodegener. 2017 Feb 20;12(1):19
59 Schaefer A, Kong R, Gordon EM, Laumann TO, Zuo XN, Holmes AJ, et al. Local-Global Parcellation of the Human Cerebral Cortex from Intrinsic Functional Connectivity MRI. Cereb Cortex. 2018 Sep;28(9):3095-114.

60 Fan L, Li H, Zhuo J, Zhang Y, Wang J, Chen L, et al. The Human Brainnetome Atlas: A New Brain Atlas Based on Connectional Architecture. Cereb Cortex. 2016 Aug;26(8): 3508-26.

61 Power JD, Cohen AL, Nelson SM, Wig GS, Barnes KA, Church JA, et al. Functional network organization of the human brain. Neuron. 2011 Nov;72(4):665-78.

62 Joliot M, Jobard G, Naveau M, Delcroix N, Petit L, Zago L, et al. AICHA: an atlas of intrinsic connectivity of homotopic areas. J Neurosci Methods. 2015 Oct;254:46-59.

63 Craddock RC, James GA, Holtzheimer PE 3rd, Hu XP, Mayberg HS. A whole brain fMRI atlas generated via spatially constrained spectral clustering. Hum Brain Mapp. 2012 Aug; 33(8):1914-28. 